\title{
Dependence of Cosmic-Ray Transport on the Orientation of the Solar Magnetic Dipole.
}

E. Antonucci

Istituto di Fisica Generale dell'Università - Torino, Italia

D. MAROCCHI

Istituto di Astronomia dell' Università - Torino, Italia

G. E. Perona

Istituto di Elettronica e Telecomunicazioni del Politecnico - Torino, Italia

(Nuovo Cimento $O, \mathbf{1}, 395(1978)$ )

The following corrections should be inserted in the above-mentioned paper:

P. 396, line 19 This 22-year cycle.

P. 396, line $34 \quad \ldots$ excursion of the Earth from $7^{\circ}$ North, on September 7, to $7^{\circ}$ South, on March $7 \ldots$.

P. 399, line 41 ... during 1954-1958 and 1970-1976 ...

P. 402, line $16 \quad \ldots$ hence $A$ is related only to the IMF polarity configuration. 